\title{
MAINTENANCE OF MALES AND FEMALES IN HERMAPHRODITE POPULATIONS
}

\author{
TAl-YING HO and M. D. ROSS \\ Department of Biology, Dalhousie University, Halifax, Nova Scotia, Canada*
}

Received 7.ix.73

\section{SUMMARY}

The effects of heterozygote advantage for fitness at a recessive male-sterility and at a dominant female-sterility locus, and the effects of linkage between these loci, were studied with reference to the maintenance of males and females in hermaphrodite populations. With some values for heterosis, and with free recombination, males, females, hermaphrodites and neuters were retained in equilibrium populations.

Linkage resulted in increased proportions of males and females, and decreased proportions of hermaphrodites and neuters. When linkage was complete neuters were eliminated. Linkage disequilibrium increased with reduced recombination.

\section{Introduction}

Among the flowering plants dioecy appears to have evolved from hermaphroditism on many occasions. Consequently, the flowering plants may prove useful in studying various stages in the evolution of dioecy. Lewis (1942) suggested that dioecy could have evolved in some flowering plants through the occurrence of two mutations, a recessive $m$ for male sterility, and a dominant $F$ for female sterility. Complete linkage between the two loci would be needed in order to prevent the generation of hermaphrodites and neuters by recombination. Thus we have the following scheme: $m f / m f q \times M F / m f \delta^{\jmath}$, giving only the parental types in the offspring. Recombinant gametes $M f, m f$ would upset the system by leading to the production of hermaphrodites, and neuters, respectively.

Lewis' scheme appears to account in outline for a possible mode of origin of dioecy, but it does not consider the process by which the sterility mutants spread through the population, ultimately displacing the wild-type alleles for fertility. If the mutants differ from wild type solely in the sterility of one sex, they will require some mechanism to maintain them and cause their spread through the population.

In this paper we adopt Lewis' model for the evolution of dioecy, and we consider the effects of heterosis and linkage on the maintenance of the maleand female-sterility mutations.

\section{MethoD}

The method is a computer simulation of a model developed by Shaw (1957) for unisexual organisms, and modified for hermaphroditic plants by Ross and Shaw (1971); fuller details of the method can be found in these publications. The model assumes random mating with no overlap of generations, and requires as input initial frequencies of the various genotypes,

* Present address: Tai-ying Ho, Dept. of Pathology, University of Toronto, Toronto 2, Ontario, Canada; M. D. Ross, Grasslands Division, D.S.I.R., Palmerston North, New Zealand.

$32 / 1-\mathrm{H}$ 
relative numbers of offspring left by each type of mating, and the genetic segregations for the mode of inheritance to be studied.

Many computer runs, using several sets of fitness values, four recombination values, and a variety of initial genotype frequencies, were made on an IBM 360/50 at Dalhousie University. All initial genotype frequencies appeared to converge to a single equilibrium for each set of fitness and recombination values. Additional runs were made on an IBM 1130 at Massey University, Palmerston North, New Zealand, using only one initial genotype frequency. Iterations were continued until equilibrium was reached except where noted.

\section{ThE SELECTION MODEL}

The 10 genotype arrangements for genes $M$ and $F$, together with phenotypes, are given at the head of table 1 . Genotypes given in parentheses there (the three homozygotes for $F$ ) do not occur, however, because they result only from impossible matings.

Fitness in this model is specified by a $10 \times 10$ array, called $W$, the elements of which represent the relative numbers of offspring left by given crosses. The values of these $W$-array elements were derived by assigning fitness values to each genotype when functioning as female (table l, first of each set of fitness values), and when functioning as male (table 1 , second of each set of fitness values). The first set of fitness values is then written on one axis of the $W$-array, the second set is written on the other axis, and the elements of the $W$-array are obtained by multiplication. In this way the elements representing like-sex " crosses" are automatically set at zero. Heterosis is then superimposed upon this basic fitness model. This method of obtaining the $W$-array is the second method of Ross and Shaw (1971).

We have used values for heterosis which are symmetrical and multiplicative between loci, e.g. when the heterozygote at the first locus has a fitness value two times that of the homozygote, then the heterozygote at the second locus is also given a fitness value of two times that of the corresponding homozygote, while the fitness value of the double heterozygote is taken as four times that of the double homozygote. Fitness values used in this study are given in table 1 .

\section{Results}

Table 2 gives the equilibrium proportions of the sex forms for varying degrees of heterosis at the sex-determining loci, and for varying amounts of recombination between these loci. The table also gives linkage disequilibrium, $D$. Where there is no heterosis, the population tends to become wholly hermaphrodite, as expected, while for the lowest heterosis value $(1 \cdot 3)$ only females and hermaphrodites are found at equilibrium. When the heterosis value reaches 2, equilibrium proportions of the various sex forms become affected by recombination frequency, and males and neuters, in addition to females and hermaphrodites, may appear in the population. With increased values for heterosis ( 3 and 10 ), and free recombination $(c=0.5)$, hermaphrodites decrease markedly, females decrease slightly, and males and neuters increase. Linkage at each heterosis value has the effect of further decreasing the proportions of hermaphrodites, reversing the increase in proportions of neuters, and increasing the proportions of males and females. Complete linkage $(c=0)$ eliminates the neuters in each case. 
NOTES AND COMMENTS

है $0+0$-0 -0 -0 -0

Z1 20000000000

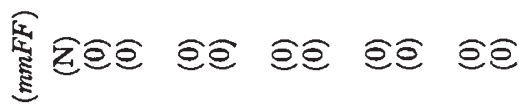

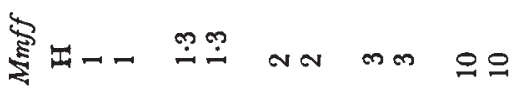

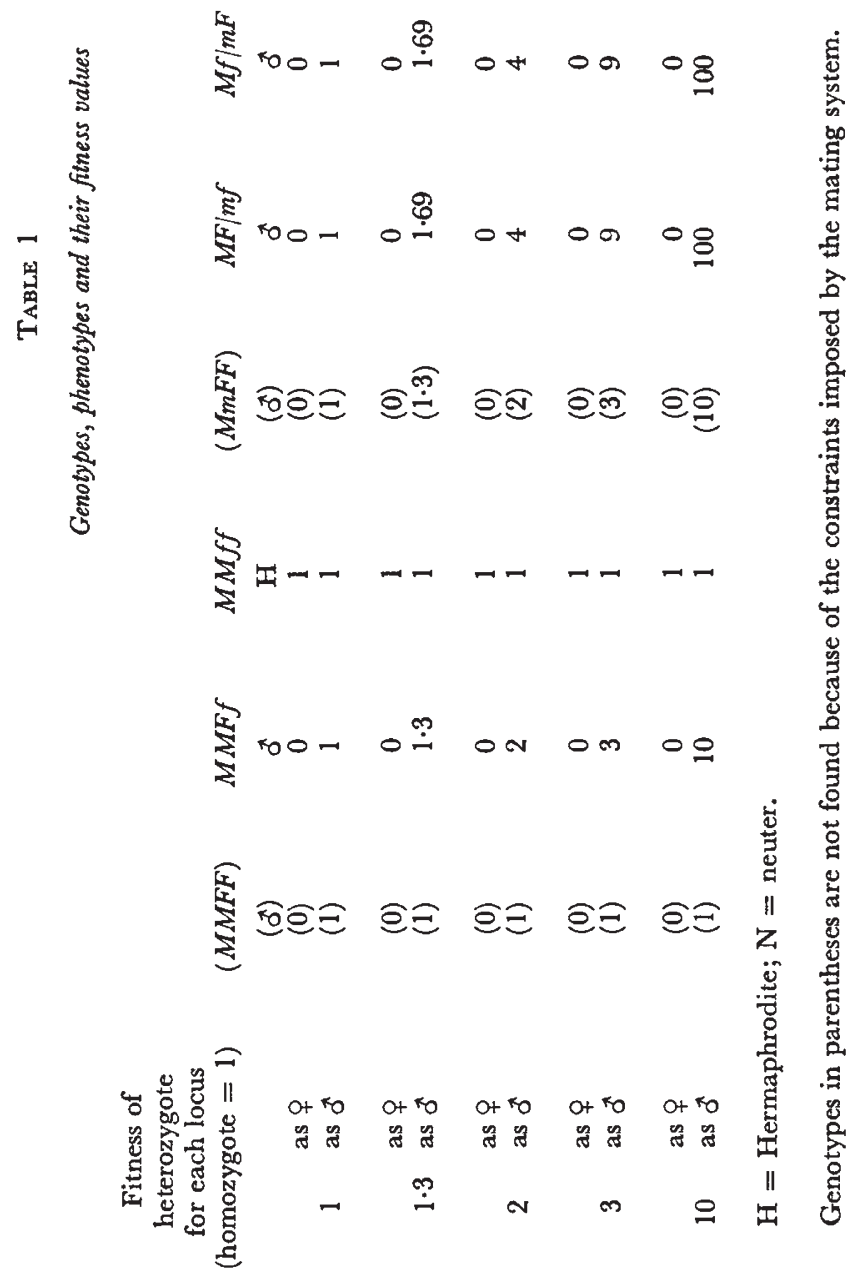

$32 / 1-\mathrm{H} 2$ 


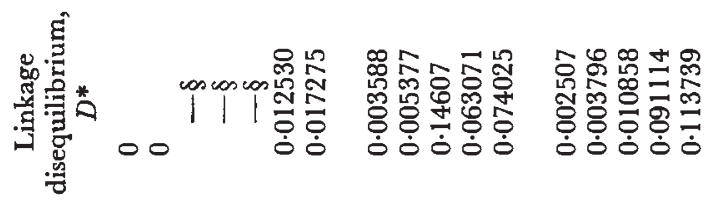

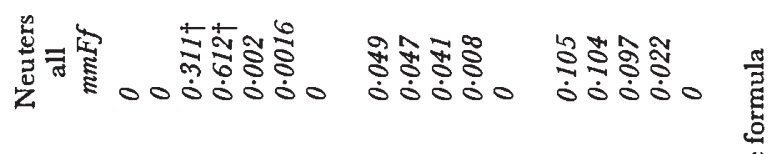

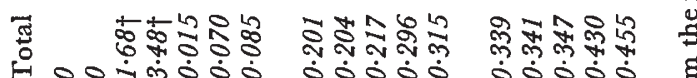

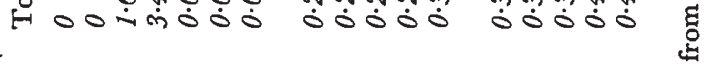

( 苞

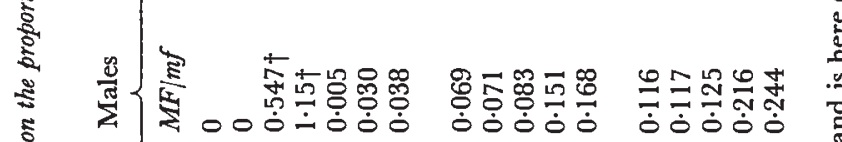

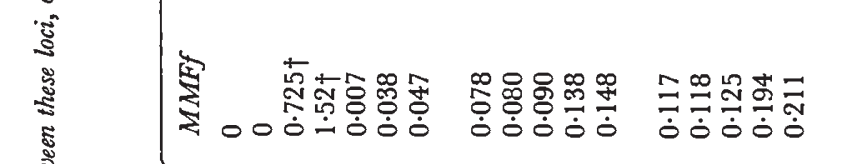

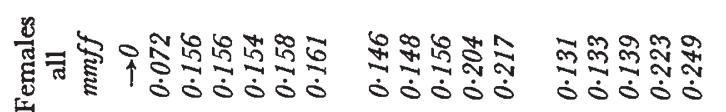

(

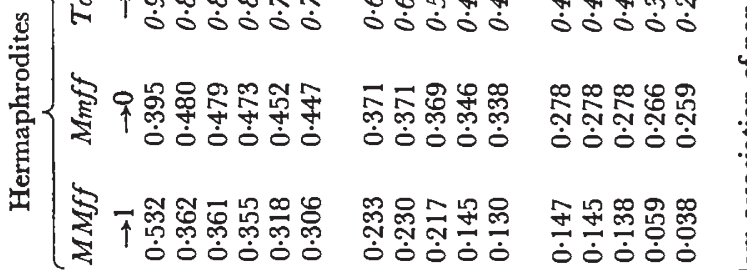


For the heterosis values 2, 3 and 10 the increased proportions of males and females which develop with reduced recombination frequencies are accompanied by increases in linkage disequilibrium, $D$. For heterosis values 3 and $10, D$ is positive even if the genes are unlinked.

In addition to presenting information regarding sex phenotypes, table 2 also contains genotype frequencies. The table shows that with increased heterosis the heterozygous hermaphrodite, $M m f f$, increases with respect to the homozygous hermaphrodite, $M M f f$, as expected. At the low hererosis value $(1 \cdot 3)$ there are no males at equilibrium, but females (genotype $\mathrm{mmff}$ ) are present. These would be produced by crosses among heterozygous hermaphrodites, Mmff. Of the three male genotypes, $M M F f, M F / m f$, $M f / m F$, the first two increase in frequency, and the third decreases, with reduced recombination; the third type is finally lost when $c=0$. At this point gamete $m F$ is lost from the population, thereby ensuring the loss of the neuters also. The first male genotype, $M M F f$, is not lost from the population under any of the conditions in the model, and would be maintained as long as hermaphrodites and males of the second genotype remain.

\section{Discussion}

We have shown that heterosis and linkage could interact to maintain males and females in initially hermaphrodite populations, where males and females are inherited by two separate mutations. Increased proportions of males and females are associated with reduced recombination and increased heterozygote advantage. Under conditions which maintain the highest proportions of males and females in the model, several features known or thought to be associated with dioecy are found. Such features are complete linkage between male-sterility and female-sterility genes, positive linkage disequilibrium, absence of repulsion heterozygotes and absence of neuters. Several other features of the model are not associated with strict dioecy. Among these are presence of hermaphrodites and presence of more than one male genotype. However, populations of subdioecious or "polygamodioecious" species may contain considerable proportions of more or less hermaphroditic individuals, together with females and males.

The fitness interactions between loci assumed in the present model are simple, but many others are of interest and have been studied theoretically (e.g. Jain and Allard, 1966). Complex fitness interactions have been observed in barley populations (Weir, Allard and Kahler, 1972), and it appears that the application of other fitness models to the present situation could be of interest.

Acknowledgments.-We wish to express our sincere thanks to Dr R. F. Shaw, of the Centre Hospitalier Universitaire, Université de Sherbrooke, for the computer method and helpful advice. Part of this work is taken from a thesis submitted by Tai-ying Ho to Dalhousie University in partial fulfilment of the requirements for the M.Sc. degree. We thank Dalhousie University Faculty of Graduate Studies for financial aid. We thank also Mr V.J. Thomas for programming assistance, and Dr B. S. Weir, Dr D. G. Lloyd and Dr E. L. Breese for reading the manuscript.

\section{REFERENCES}

JAIN, s. K., AND ALlARD, R. w. 1966. The effects of linkage, epistasis, and inbreeding on population changes under selection. Genetics, 53, 633-659.

LEWIS, D. 1942. The evolution of sex in flowering plants. Biol. Rev., 17, 46-67. 
ROSs, M. D., AND SHAW, R. F. 1971. Maintenance of male sterility in plant populations. Heredity, 26, 1-8.

SHAW, R. F. 1957. The theoretical genetics of the sex ratio. Genetics, 43, 149-163.

WEIR, B. s., ALLARD, R. W., AND KAHLER, A. L. 1972. Analysis of complex allozyme polymorphisms in a barley population. Genetics, 72, 505-523.

\title{
GIEMSA STAINING AND THE DISTRIBUTION OF HETEROCHROMATIN IN RYE CHROMOSOMES
}

\author{
S. C. VERMA and H. REES \\ Department of Agricultural Botany, University College of Wales, Aberystwyth
}

Received 12.ix.73

\section{SUMMARY}

$C$-banding, by Giemsa staining, is largely restricted to distal regions of rye chromosomes. This applies, also, to B chromosomes. The distribution of C-bands coincides with that of distally localised heterochromatin. The area of metaphase chromosomes staining with Giemsa corresponds to the area occupied by heterochromatin in interphase nuclei as revealed by Feulgen staining. The Giemsa-stained $C$-bands account, therefore, for all the heterochromatin in rye nuclei. Individual chromosomes within the complement are readily identified following Giemsa staining.

\section{INTRODUCTION}

Giemsa-staining methods developed, initially, by Pardue and Gall (1970) have revealed hitherto undetectable and indeed unsuspected patterns of structural differentiation in metaphase chromosomes. The following account describes how Giemsa staining in rye may be used to identify individual chromosomes within the complement and, as well, to map the distribution of heterochromatin within the chromosomes.

\section{Materials AND MEthods}

The rye plants used were from a population carrying $B$ chromosomes, the $\mathrm{B}$ frequency ranging from 0 to 8 per plant. The population was derived initially from crosses between plants of Secale cereale, of the variety Stälrag, and S. vavilovii plants carrying B's (see Jones and Rees, 1967). We could detect no morphological difference between the chromosome complement of this population and those of "pure" $S$. cereale populations. The reason for choosing this particular population was that it contained many B's.

Seeds were germinated on moist filter paper in petri dishes in the dark at $20^{\circ}$ C. Roots from seedlings with three to five roots were immersed in 0.2 per cent colchicine for 5 hours and fixed, subsequently, for 4 hours in 1:3 acetic acid : absolute alcohol. The Giemsa-staining procedure used was that devised by Vosa and Marchi (1972) for plant material. The technique was as follows:

1. Hydrolysis in $1 \mathrm{~N} \mathrm{HCl}$ at $60^{\circ} \mathrm{C}$. for 25 seconds, followed by thorough washing in distilled water. Further softening of root tips by immersion in 45 per cent acetic acid. 